\title{
BMJ Open Use of virtual and augmented reality- based interventions in health education to improve dementia knowledge and attitudes: an integrative review
}

\author{
Cindy Jones, ${ }^{1,2}$ Dominique Jones, ${ }^{1}$ Christian Moro (D) ${ }^{1}$
}

To cite: Jones C, Jones D, Moro C. Use of virtual and augmented reality-based interventions in health education to improve dementia knowledge and attitudes: an integrative review. BMJ Open 2021;11:e053616. doi:10.1136/ bmjopen-2021-053616

- Prepublication history and additional supplemental materia for this paper are available online. To view these files, please visit the journal online (http://dx.doi.org/10.1136/ bmjopen-2021-053616).

Received 19 May 2021 Accepted 11 October 2021
D) Check for updates

(c) Author(s) (or their employer(s)) 2021. Re-use permitted under CC BY-NC. No commercial re-use. See rights and permissions. Published by BMJ.

${ }^{1}$ Faculty of Health Sciences and Medicine, Bond University, Robina, Queensland, Australia ${ }^{2}$ Healthcare Practice and Survivorship, Menzies Health Institute Queensland, Healthcare Practice and Survivorship, Brisbane, Queensland, Australia

Correspondence to Dr Christian Moro; cmoro@bond.edu.au

\section{ABSTRACT}

Objectives Immersive technologies such as virtual (VR) and augmented reality (AR) can potentially help health professionals and trainees understand psychological symptoms and responsive behaviours associated with dementia within a safe and supportive learning environment. This integrative review sought to ascertain the types of VR and AR-based interventions used in dementia education and training and its efficacy to improve knowledge and attitudes of health professionals or trainees.

Design The protocol was submitted to PROSPERO and literature published from 2000 onwards was searched in eight databases: CINAHL, MEDLINE, Web of Science, Cochrane, Embase, PsycINFO, ERIC and Scopus. A total of 19 articles were included and assessed with the Mixed Methods Appraisal Tool. Methodological quality varied across studies.

Results VR rather than AR-based intervention are used in dementia education and training for health professional and trainees. Immersive virtual learning potentially enhance knowledge, attitudes, empathy and sensitivity of health professionals and trainees.

Conclusions While promising, there remains a lack of conclusive and robust evidence to fully recommend the introduction and inclusion of immersive virtual learning in dementia education and training. Additional rigorously designed research studies with larger sample sizes are needed to confirm the benefits on attitudes, empathy, sensitivity and knowledge.

PROSPERO registration number CRD42020182083.

\section{INTRODUCTION}

Education and training of health professionals and trainees (ie, individuals formally trained or training to work in a health or healthrelated field), such as those in the fields of medicine and nursing, conventionally adopt the coaching approach of 'see one, do one, teach one'. ${ }^{2}$ However, the growing emphasis on patient safety, care and awareness, as well as an increasing teaching cost in the clinical environment have led to the consideration and incorporation of advanced technology in coaching techniques. ${ }^{3}$ One of these is
Strengths and limitations of this study

- This is the first review to assess the use of virtual and augmented reality in dementia education and training across both qualitative and quantitative studies.

- This review conducted a quality assessment of the 19 included studies.

- The 19 included studies reported a broad range of sample sizes and reporting metrics, resulting in difficulties for formal comparisons and clear recommendations.

- Outcomes of this review may be subjected to selection bias due to the exclusion of grey literature and the inclusion of only studies published in English.

immersive technologies that are defined as 'devices that provide sensory stimuli to provide a sense of realism and immersion to the interactions with the computer-generated world'. ${ }^{4}$ Virtual reality (VR) and augmented (AR) are two principal types of immersive technologies. In the last 10 years, VR and AR have evolved from what was previously considered futuristic technology in science fiction to the reality of a potentially effective tool for health-related educators. ${ }^{5-7}$

Virtual experiences can exist in various forms (ie, fully, semi or non-immersive). Nonimmersive virtual experiences are common in daily life. It refers to computer-generated environment where the user remains perceptive and maintains control of the physical environment. One such example is video games or activities, using consoles, keyboards, mice and controller. VR is regarded as a fully immersive experience or a computer simulated three dimensional (3D) world experience, where the user can interact with the virtual environment and characters via special electronic devices such as an enclosed headset or body suit. ${ }^{689}$ Users of VR devices (eg, Oculus Quest/Rift or HTC Vive) are fully immersed 
in a completely enclosed digital environment with sight and sound but no sense of the real world. On the other hand, $\mathrm{AR}$ is an enhanced reality or a semi-immersive virtual experience where realism is provided through 3D graphics, also known as vertical reality depth. The user operates an application on a device such as a smartphone, to render an interactive digital layer or image on top of reality (eg, a physical object). ${ }^{5610}$ Experience of the real world remains central to users of AR but this experience is enriched by information provided through a digital virtual overlay. With the rapid advancement and popularity of VR and AR, innovative ways to leverage immersive technologies are anticipated to transform not only healthcare education and training but also practices in areas of treatment and therapies (eg, in surgery), telemonitoring, care planning as well as patients' experience. ${ }^{11} 12$ Specifically, this type of modern technology may influence the way education and training for dementia care and awareness could be effectively delivered in the future.

The WHO recognises dementia as a public health priority, and places an emphasis on enhancing health professionals' awareness of the condition with the goal to improve care and support for people living with dementia. ${ }^{13}$ The need for better ways to educate our health professionals on dementia is essential now more than ever, as there are currently an estimated 50 million people living with the disease globally, with this expected to increase threefold to 115.4 million by $2050 .{ }^{13-15}$ As health professionals and trainees would have never personally experienced life from the perspective of someone with dementia, ${ }^{14} 16$ and some may not have prior personal or work experience in interacting or caring for people living with dementia, many have expressed feeling underprepared to manage and care for them. ${ }^{17-19}$ For this reason, the nature and approach employed when determining which form of dementia training to use is an important area for consideration in health education.

Immersive technologies such as $\mathrm{VR}$ and $\mathrm{AR}$ can provide a feasible mean for orientating health professionals and trainees to aspects of dementia (eg, psychological symptoms and responsive behaviours related to dementia) that can be confronting but relevant to their work roles within a safe and supportive learning environment. ${ }^{20}{ }^{21}$ Responsive behaviours (eg, those brought on by unmet needs or a lowered stress response threshold) are displayed by up to $90 \%$ of people living with dementia in the form of agitation, aggression, apathy, repeatedly calling out, sleepdisturbance and wandering ${ }^{22}$ and have been reported to be distressing or challenging for health professionals. ${ }^{21}$ Those who have limited understanding of the causes of these behaviours can misunderstand their origins and the person's related intentions, potentially lowering the quality of care provision. ${ }^{21}$ As such, it is imperative for health professionals to have the requisite education and training to respond to these complex psychological symptoms and responsive behaviours of dementia in an empathetic, patient and understanding manner. ${ }^{141623}$ Over the years, there have been growing utilisation of high-quality immersive technologies such as VR and AR in dementia education and training to enable health professionals and trainees to explore what and how it feels to be experiencing dementia, that is to see the world through the eyes of a person living dementia. More importantly, it can potentially improve their dementia knowledge and enhance their understanding of caring for someone living with dementia. This may then allow for them to identify support needs and develop a supportive approach to assist people living with dementia to live confidently and independently. Therefore, this review aims to examine current evidence of what and how VR and AR interventions are being used in dementia education and training as well as their efficacy to improve knowledge and attitudes of health professionals and trainees towards people living with dementia.

\section{METHODS}

\section{Aim}

Published literature, both qualitative, quantitative and mixed-methods studies, on VR and AR-based dementia training programmes is described, evaluated and synthesised in this review to answer the following research questions:

1. What are the different types of VR and AR-based interventions used in dementia education and training for health professionals or trainees?

2. What is the efficacy of health education interventions involving VR and AR in improving dementia knowledge and attitudes of health professionals or trainees?

\section{Protocol}

The Preferred Reporting Items for Systematic Reviews and Meta-Analyses (PRISMA) statement was followed for this review's outline. ${ }^{24}$ The protocol was developed prospectively and submitted to PROSPERO, an International Prospective Register of Systematic Reviews ${ }^{25}$ on 24 April 2020. This protocol was followed with only very minor deviations where required, and these are reported in the Methods section.

\section{Design}

An integrative approach is adopted where study outcomes from various methodologies and data are included to provide a comprehensive understanding of the phenomenon of interest. ${ }^{26}$ Utilisation of this approach is suitable for this review as it will ensure the provision of an all-encompassing overview and contribution to the body of knowledge, practice and research in relation to VR and AR-based interventions used to educate or improve health professionals or trainees on dementia. ${ }^{26}$

\section{Search strategy}

Eight electronic databases across health, science, psychology, medicine and education were searched: CINAHL (EBSCO), MEDLINE (Ovid), Web of Science Core Collection, Cochrane Central Register of Controlled Trials 
(CENTRAL; Wiley), Embase (Elsevier), PsycINFO (Ovid), ERIC (Ovid) and Scopus. Articles published between 2000 and 2021 were identified using the following search terms: "virtual realit*" OR VR OR "augmented realit*" OR AR OR "mixed realit*" AND health AND intervention OR educat* AND dementia OR "Alzheimer disease" AND knowledge* OR attitude* OR experience* OR aware* OR understand* OR perception* OR comprehension* AND "health professional*" OR personnel* OR "allied health personnel" OR doctor* OR "medical staff" OR "nurs* staff" OR "physical therap*" OR physiotherapy OR "occupational therap*" OR dieti* OR trainee* OR student*. The mesh terms included were 'virtual reality', 'augmented reality', 'mixed reality', mixed realities', 'augmented realities', 'health', 'education', 'training', 'dementia', 'Alzheimer disease', 'knowledge', 'attitude', 'awareness', 'understanding', 'perception', 'comprehension', and 'allied health personnel'. The Polyglot tool ${ }^{27}$ was used to check title, abstract and keyword search terms. Reference lists of eligible articles and systematic reviews pertinent to this research area were searched manually. Full search strategies for all databases are presented in online supplemental file 1 .

\section{Inclusion/exclusion criteria}

To be included in this review, articles had to meet the following criteria: (i) primary full-text studies published in English; (ii) published between 1 January 2000 to 31 March 2021; and (iii) qualitative, quantitative and mixedmethods studies involving the use of VR and AR-based interventions to improve dementia knowledge and attitudes of health professionals or trainees. Articles that reported: (i) trial registration materials; (ii) studies involving non-health professionals or trainees and (iii) non-original research such as literature reviews, theses, newsletters, editorials, commentaries, discussion papers and notes were excluded. Conference proceedings were also excluded with the exception of full text, peerreviewed conference papers.

\section{Search outcome}

A list of 97 records was yielded from the initial search from databases. Following the removal of duplicates $(n=17)$, title and abstract screening of 80 records plus a further 17 articles, identified through manual searching, were conducted independently by two reviewers (CJ and DJ) in line with the inclusion and exclusion criteria. A total of 31 records were eligible for full-text assessment. The full texts of these records were retrieved and assessed independently against the inclusion and exclusion criteria by the same two reviewers (CJ and DJ). No disagreement occurred in the article selection process where a total of 19 articles were identified to be eligible for inclusion in this review (refer to figure 1). Figure 1 presents the PRISMA flowchart diagram that illustrates the literature search and selection process. ${ }^{24}$

\section{Quality appraisal}

The Mixed Methods Appraisal Tool (MMAT) Version 2018 was used to assess the quality of the 19 included articles. ${ }^{28}$
The MMAT is selected as it includes two initial questions to screen for empirical studies. Once established, it can be used to efficiently appraise different study designs (eg, quantitative randomised and non-randomised controlled trials, quantitative descriptive studies, qualitative studies and mixed-methods studies) across core quality criteria with the outcome for each criterion reported descriptively. ${ }^{29}$ Two reviewers (CJ and DJ) conducted the quality appraisal independently. Outcomes of the quality assessment were cross-checked. A 5\% disagreement found was subsequently resolved via referral to the third reviewer (CM) to reach quality appraisal consensus for all included studies.

Data extraction from the 19 included articles was initially completed by one reviewer (DJ) and then checked by a second reviewer (CJ). Information extracted included authors, publication year, country, study aim(s), participants, study design, intervention, outcome measures, results and study limitations including quality appraisal (refer to online supplemental file 2). An integrative approach, which involves the reduction, display and comparison of data followed by the drawing and verification of conclusions, ${ }^{26}$ was used to synthesise data from all included studies. This was performed by two reviewers separately (CJ and DJ) to avoid bias. Studies included in the review are organised into groups and subgroups for the purpose of data reduction followed by arrangement of data in a way that allows the visualisation of patterns, relationships and variation to support the iterative analysis process. Conclusions are then made and verified to ensure the depth and breadth of included studies are evidently presented. Evidence gathered from the review, on interventions involving $\mathrm{VR}$ and $\mathrm{AR}$ in improving dementia knowledge and attitudes of health professionals or trainees, is summarised and presented narratively to identify any knowledge gaps and highlight areas for future research.

\section{Patient and public involvement}

No patients or the public were involved in the design, conduct, reporting or dissemination plans of our research.

\section{RESULTS}

\section{Study characteristics and participants}

In the 19 included articles, the earliest published paper was in 2002. ${ }^{16}$ Studies in this review were conducted in Australia, ${ }^{81830}$ China, ${ }^{31}$ Ireland, ${ }^{32}$ Korea,,${ }^{14} \mathrm{UK}^{1720}$ and the USA. $^{141633-41}$ Of these studies reviewed, seven were quantitative (ie, non-randomised controlled and pre-quasiexperimental and post-quasi-experimental studies with one or two groups), ${ }^{8161834363740}$ five qualitative (ie, exploratory or phenomenology studies via reflection, individual or focus group interview), ${ }^{14} 30324142$ and seven mixed methods (ie, quasi-experimental studies with pretest and post-test plus reflection or interview). ${ }^{17203133353839}$ Across the studies reviewed, most participants were undergraduate students or students studying a discipline related 


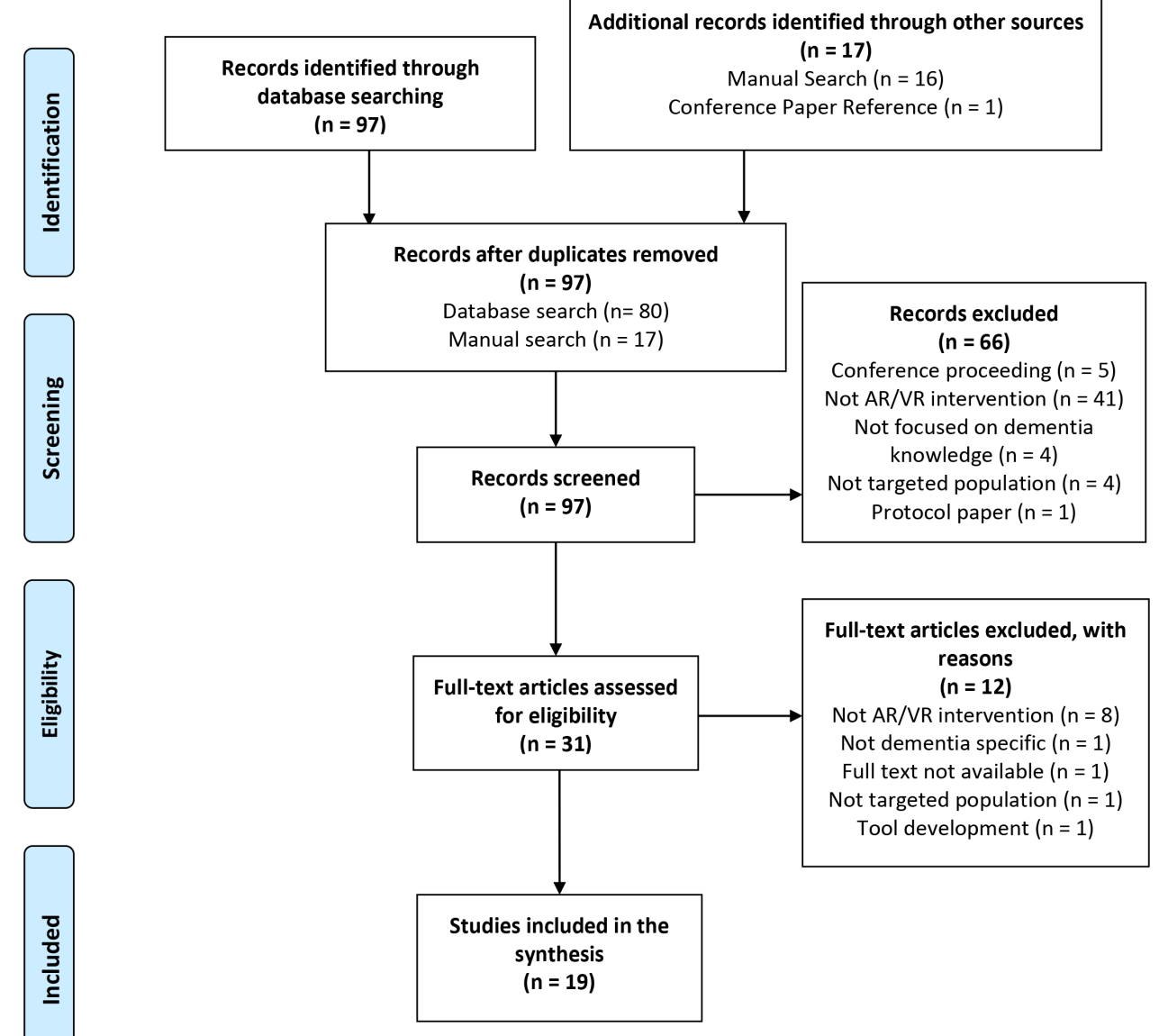

Figure 1 Preferred Reporting Items for Systematic Reviews and Meta-Analyses 2020 flow diagram. AR, augmented reality; VR, virtual reality.

to health (ie, medicine, nursing, paramedics, pharmacy, psychology, occupational or physical therapy), social work or human services. ${ }^{8} 171820303133-42$ Other participants include healthcare workers (ie, registered nurses, allied health professionals, social workers care aides/assistants, administrative and management staff including directors of nursing) and formal and informal caregivers ${ }^{141632}$ with one study involving health faculty teaching staff members. ${ }^{41}$ As reflected in online supplemental file 2, not all studies reported participants' gender and age but of those that did, majority of participants were females and young adults.

\section{VR/AR interventions and outcomes}

A variety of interventions were assessed across the included studies. The intervention used in the majority of articles reviewed (ie, 10 out the 19) was the Virtual Dementia Tour (VDT). ${ }^{16}$ Beville $^{16}$ designed and created a VDT experience, a dementia sensitivity training programme, that altered users' senses when completing everyday tasks. Users of the VDT were 'garbed' in special attire (ie, patented devices) to stimulate senses (eg, 'placing popcorn kernels in shoes/gloves to simulate poor circulation, neuropathy and/or arthritis as well as the loss of sensory and motor skills') in a distorted environment (eg, 'goggles darkened with yellow cellophane, smeared with Vaseline and a centred black dot to simulate variety of visual impairments') and were required to complete five simple tasks (eg, find and put on a sweater). ${ }^{43}$ Studies involving the VDT either replicated it as the sole intervention $^{1632} 33353641$ or combined it with other activities such as dementia-related lecture or movie/video, clinical experience, case vignette, role-play and/or discussion. ${ }^{3134} 3940$

Four articles evaluated the experiences of users who completed the Dementia Live (DL) programme. ${ }^{14} 373842$ This is a programme where users immersed in a simulated life of living with dementia and tasked to complete daily activities (eg, sorting cutlery) while experiencing cognitive impairments and sensory losses that are induced through headphones with MP3 Player (to distract attention and hearing), eyewear (to limit peripheral vision) and gloves (to weaken the sense of touch and fine motor skills). ${ }^{44}$ The DL programme was modified for use in a Korean cultural context $^{14}$ and in conjunction with dementia-related lecture. $^{38}$

Of the remaining five articles, two reported on the same myShoes project intervention study where one is a conference paper ${ }^{20}$ and the other is a research article. ${ }^{17}$ The myShoes project involves the augmentation of a 
virtual environment to increase users' understanding of the experience of living with dementia. ${ }^{17}$ Through the use of Oculus Rift (ie, a stereoscopic headset with touch controllers) and the Unity 3D software, users immerse in a manipulated virtual environment, aimed at causing misdirection and confusion, and navigate the avatar of an older person to complete simple tasks (eg, clearing dining table). Users will experience the emotions and cognitive function impairments people living with dementia encounter. Another two articles $^{18} 30$ reported on Alzheimer's Australia Victoria Virtual Dementia Experience (VDE), where an immersive multisensory, virtual simulation of light, sound, colour and visual content is projected on a $10 \times 2 \mathrm{~m}$ screen, aimed for users to experience cognitive and perceptual difficulties encountered by people living with dementia. The final article on the Virtual Reality Educational project ${ }^{8}$ conducted an intervention using the Oculus Rift headset and sensor, Leap Motion handtracking device and Embodied Labs programme for users to experience ageing-related macular degeneration and high-frequency hearing loss as well as conversations relating to Alzheimer's disease and end-of-life.

Studies reviewed had a focus on improving participants': (i) knowledge of dementia ${ }^{183436-40}$; (ii) attitudes towards dementia including perceived confidence and competence in care provision ${ }^{17} 18203436373940$; (iii) empathy for people living with dementia ${ }^{817203137-40}$ and/or (iv) understanding and awareness of people living with dementia and sensitivity towards their care needs. ${ }^{1633}$ Instruments used to measure outcomes varied across studies. Knowledge and attitudes were measured using the Knowledge About Memory Loss and Care, ${ }^{45}$ Dementia Knowledge Assessment Tool Version 2, ${ }^{46}$ Knowledge in Dementia Scale, ${ }^{47}$ Dementia Attitudes Scale,${ }^{48}$ or Confidence in Dementia Scale. ${ }^{47}$ While sensitivity was assessed using the survey developed by Beville ${ }^{16}$ for the VDT, empathy was measured using the Interpersonal Reactivity Index (IRI) ${ }^{49}$ Comprehensive State Empathy Scale ${ }^{50}$ or Jefferson Scale of Empathy-Health Profession Students. ${ }^{51}$ These outcomes, together with perceived intervention experience, were also assessed qualitatively through individual and focus group interviews, observations and written reflections.

\section{Knowledge and attitudes}

The impact of interventions involving VR and AR on dementia knowledge is mixed. Of the seven studies evaluating dementia knowledge, four found a significant improvement in participants' knowledge of dementia from preintervention to postintervention when using the VDE, ${ }^{18} \mathrm{DL}^{37}$ and VDT combined with dementia video, case vignette and role-play. ${ }^{39} 40$ However, where studies involved a two-group comparison, ${ }^{37} 40$ no significant group difference on dementia knowledge was found. The remaining three studies using DL with dementia lecture ${ }^{38}$ and VDT, ${ }^{34} 36$ where one also included dementia lectures, ${ }^{34}$ did not find any improvement in participants' knowledge of dementia. On the other hand, significant improvements in attitudes towards dementia including perceived confidence and competence in care provision were found across all seven studies and intervention types that assessed attitudes, confidence and competence. ${ }^{17} 18203436373940$ Likewise to knowledge, no group difference on attitudes was found in studies with a two-group comparison. ${ }^{18} 3740$ Qualitative data indicated that participants did perceive an overall improvement in their knowledge and attitudes towards people living with dementia, ${ }^{30}$ as well as knowledge and confidence in dementia care following their intervention experience. ${ }^{39}$

\section{Empathy and sensitivity}

All studies, except for one using DL, ${ }^{40}$ that focused on empathy reported a significant improvement from preintervention to postintervention. myShoes, ${ }^{1720}$ Virtual Reality Educational (VRE), ${ }^{8}$ DL with and without ${ }^{37} 38$ dementia lecture as well as VDT combined with dementia video, case vignette and role-play ${ }^{39}$ or movie ${ }^{31}$ led to significantly enhanced empathy in participants. However, no difference in empathy was found in Kimzey et $a l \mathrm{~s}^{37}$ two groups study. Furthermore, both studies by Kimzey et $a l^{37}$ and Mastel-Smith $e t a l^{39}$ found an increased tendency in participants to spontaneously adopt the psychological point of view of people living with dementia (from IRI subscale) following VR and AR interventions. Participants described the VR and AR intervention as enlightening as they were able to gain an appreciation and comprehend that living with dementia is much harder than anticipated and could imagine how a person living with would feel and behave (empathic imagination). ${ }^{38} 42$ They reported understanding and sharing feelings of distress (eg, sadness, anxiety, fear, frustration, anger and helplessness) experienced by people living with dementia in how they perceived the world and their future as well as when completing tasks related to daily activities of living. ${ }^{14} 31323839$ Importantly, the opportunity of a simulated lived experience of living dementia enables reflection on a cognitive, moral and behavioural level, leading to empathy and promoting the use of new helpful care strategies. ${ }^{32} 4142$

For VDT studies that assessed understanding and awareness of people living with dementia and sensitivity towards their care needs using the VDT survey, the use, analysis and results reporting of the survey varied across studies. ${ }^{1633-36}$ Nevertheless, all studies reported an overall improvement (via either statistical significance indicating higher quality evidence ${ }^{33-35}$ or graphical means trend ${ }^{16} 36$, but specifically in participants' awareness of the need to be sensitised to people living with dementia's emotional needs (including feelings of anxiety) to provide good care ${ }^{16} 333^{36}$; and understand their incapability to perform simple everyday tasks, ${ }^{163}$ experience of functional difficulties and challenges to get through the day, ${ }^{1633436}$ and manifestation of inappropriate (or possibly justified) 
behaviours, ${ }^{16} 34$ as well as not always receiving the requisite care they need. ${ }^{163336}$

\section{User experience}

Generally, participants reported satisfaction and perceived usefulness and effectiveness of their VR and AR experience and would recommend it as part of professional training and development for carers of people living with dementia. $^{31} 3242$ It is considered by participants to be a viable training approach ${ }^{42}$ that addresses gaps in traditional teaching methods and provides opportunities for immersive simulated experiential learning to occur. ${ }^{31} 41$ On reflection, participants believed that these experiences have a positive impact on them both personally and professionally $^{33}$ as they give them an insight into the lives of older people living with ageing and dementia-related health challenges. ${ }^{81433}$ Participants indicated their experience has highlighted the need for them to (i) provide people living with dementia with more attention and affection; (ii) improve communication approach using dementia-friendly techniques; (iii) adopt appropriate care strategies such as not asking them to complete multiple tasks and providing ample time for tasks completion; (iv) avoid imposing negative behavioural judgements and (v) the need to support family carers. ${ }^{14}$ 30-32 3536384142

\section{Quality appraisal}

All studies met the first two screening criteria for MMAT except for the study by Dyer $e t a l^{8}$ where there was limited information provided in the rationale and description of the methodology used as well as the discussion of results. All five qualitative studies ${ }^{1430324142}$ met the quality appraisal criteria where the qualitative approach undertaken and data collected were appropriate to answer the research question. Furthermore, interpretation of qualitative findings was adequately derived and substantiated from collected data. On the other hand, none of the quantitative and mixed-method studies met all the quality appraisal criteria. One or more concerns were noted across these studies.

First, in some studies, participants without follow-up (ie, post) data were either excluded from the analysis and reasons for missing data were not always reported. ${ }^{18} 353739$ This can lead to non-response bias during the interpretation of results. Second, methodology was not clearly reported in two studies (ie, myShoes ${ }^{17}$ and $\mathrm{VDT}^{16}$ ) where there were no to limited information on study design, sampling strategy, non-response bias and/or outcome measurements used. Third, several studies were limited by its small sample size s $^{17031343839}$ and the nonreporting of demographic characteristics such as age and gender. $^{81617203640}$ Fourthly, although the VDT survey ${ }^{16}$ has been used to assess understanding and awareness of people living with dementia and sensitivity towards their care needs following a VDT experience, ${ }^{1633-37}$ there is limited empirical evidence on the face validity and internal consistency of the VDT survey. It is also questionable whether the assessment tool (ie, KIDS) used in Lorio et al.'s ${ }^{34}$ study is sensitive to changes in dementia knowledge. Finally, there was limited integration of the quantitative results and qualitative findings when interpreting and discussing the outcomes of the mixed-method study of Peng et $a l^{31}$

\section{DISCUSSION}

The literature included in this integrative review presented five types of immersive VR-based interventions used in dementia education and training for health professionals or trainees to deliver simulated cognitive, sensory and perceptual experiences similar to those when living with dementia. No AR-based intervention was identified in this review. Both the VDT and DL programmes are similar to each other, through their use of sensory devices such as gloves, goggles and headphones as well as verbal instructions for tasks completion. In contrast, myShoes and the VRE projects require the use of the Oculus Rift headset while the VDE simply projects multisensory (ie, light, sound, colour and visual) content on a large screen. The majority of published studies focused on VDT, followed by DL with a few empirical studies on myShoes, VRE and VDE. Adverse experience of VR motion sickness has been reported by some users in myShoes study ${ }^{1720}$ but not the VRE project. ${ }^{8}$ Dementia education and training using VR that incorporate VR headset needs to consider motion sickness (ie, cold sweats, vomiting and feelings of nausea and dizziness), a well-known, common occurrence in users of $\mathrm{VR}^{95253}$ as it can potentially impede on the development and adoption of this technology in future dementia education and training. However, advances in available consumer technologies, such as increased frames-per-second and movement sensors within the devices may alleviate these adverse health effects in the near future.

Earlier empirical studies have found a positive pedagogical value in immersive virtual learning when compared with traditional teaching approaches. ${ }^{9} 54-56$ There is also research evidence to demonstrate the positive impact of immersive virtual learning in health sciences and medical education and training. ${ }^{75758}$ The immersive VR-based interventions in this review are reported to have allowed users to become more knowledgeable about the dementia condition; develop more positive attitudes towards people living with dementia; feel more confident and competence in care provision; as well as being more empathetic and sensitive towards people with dementia and their care needs. These were achieved through the manipulated virtual environment that simulated dementia-related living conditions such as cognitive impairments, sensory and perceptual losses and difficulties for users; as well as from their reflections on the impact of the immersive and interactive experiences. Further benefits users gained through their participation include: learning new approaches to care for people living with dementia; environmental issues that impede on a person with dementia's daily 
routine; and environmental adaptions to address these issues to enhance care provision.

Interestingly, unlike a previous study by Webster ${ }^{59}$ which found immersive virtual learning to be as effective, if not better, than conventional lecture-based learning, this review noted that the effects of immersive virtual learning in the acquisition of declarative knowledge about dementia is conflicting and less evident when compared with improvements in attitudes, empathy and sensitivity. However, this may be explained by the purpose and nature of the immersive virtual learning where it is focused less on conceptual, propositional or descriptive content but more on users having first-hand, direct experience of dementia and ageing associated cognitive, sensory and perceptual challenges in actual daily living. The intent is placed on experiential learning where users transformed experience into an increased understanding and awareness of what is like living with dementia leading to enhanced attitudes and increased empathy and sensitivity to positively influence their care provision. ${ }^{60}$

\section{Strengths and limitations}

To our knowledge, this is the first integrative review that examines current quantitative and qualitative evidence of what and how VR and AR interventions are being used in dementia education and training as well as its efficacy to improve knowledge and attitudes of health professionals and trainees towards people living with dementia. The use of defined inclusion/exclusion criteria, rigorous search from eight databases and validated MMAT to ascertain quality of studies are considered the strengths of this review. Even though this review offers a descriptive results overview of the different types of immersive VR-based interventions used in dementia education and training as well as its efficacy on knowledge, attitudes, empathy and sensitivity of health professionals and trainees, findings should be interpreted with caution due to several limitations.

First, the small number of studies conducted for each type of reported immersive virtual learning, except for VDT, found in this review reflects the paucity of empirical studies in the research field of immersive technologies (eg, VR and AR) in dementia education and training. Second, the nature of the study designs adopted in the quantitative and mixed-method studies reviewed, as previously noted, does not permit the establishment of efficacy (ie, lack of causality due to non-control group comparison $^{816} 17203133-363839$ or non-randomisation between groups ${ }^{183740}$ ). Third, the sample sizes were quite small, and non-reporting of demographics occurred within some studies. In addition, the gender and age imbalance across studies where the majority of participants were female and young adult, together with the quality shortcomings determined through the reported methodology of included studies, suggest the need for caution in the interpretation and generalisability of findings. Fourth, the heterogeneity of study designs, immersive virtual learning (ie, types and duration), together with different types of outcome assessment and the different tools used to assess these outcomes make it impractical for results of studies reviewed to be collated for auxiliary analysis. Finally, language bias should be considered because only studies published in the English language were selected, thereby omitting the possible inclusion of studies published in other languages. Further and more rigorous empirical research with larger sample sizes is needed for the different types of immersive virtual learning used in dementia education and training to better ascertain its efficacy on knowledge, attitudes, empathy and sensitivity of health professionals and trainees.

\section{CONCLUSION}

This integrative review syntheses evidence from both quantitative and qualitative studies with a total of 19 published articles included in the review. The results imply that currently, VR, rather than AR-based interventions are used in dementia education and training. Of these, five separate studies assessing immersive VR-based interventions were identified. The VDT is the most commonly used intervention followed by the DL programme. To date, published literature suggests that immersive VR-based interventions provide a promising way for educators to enhance knowledge, attitudes, empathy and sensitivity of health professionals and trainees. However, there remains a lack of conclusive and robust evidence to fully recommend the introduction and inclusion of immersive virtual learning in dementia education and training. Additional rigorously designed RCT/CT studies with larger sample sizes are needed to confirm the benefits found in this review. In particular, the potential of immersive virtual learning to improve attitudes, empathy, sensitivity and especially knowledge requires further investigation.

\section{Twitter Christian Moro @physiologywith}

Acknowledgements Our thanks to the Faculty of Health Sciences and Medicine Librarian, Sarah Bateup, at Bond University for her assistance and guidance in the literature search.

Contributors The authors confirm contribution to the paper as follows: study conception, planning, and design: CJ, CM, DJ. Data collection: CJ, DJ. Analysis and interpretation of results: CJ, DJ, CM. Draft manuscript preparation: CJ, DJ, CM. All authors (CJ, DJ, CM) provided substantial contributions to the work, were involved in drafting, the final reporting, and approvals. Overall, CJ agrees to be the guarantor and accepts full responsibility for the work.

Funding The authors have not declared a specific grant for this research from any funding agency in the public, commercial or not-for-profit sectors.

Competing interests None declared.

Patient consent for publication Not applicable.

Ethics approval Ethical approval from the institutional Human Research Ethics Committee was not required for this integrative review.

Provenance and peer review Not commissioned; externally peer reviewed.

Data availability statement Data are available upon reasonable request. All data relevant to the study are included in the article or uploaded as supplementary information.

Supplemental material This content has been supplied by the author(s). It has not been vetted by BMJ Publishing Group Limited (BMJ) and may not have been 
peer-reviewed. Any opinions or recommendations discussed are solely those of the author(s) and are not endorsed by BMJ. BMJ disclaims all liability and responsibility arising from any reliance placed on the content. Where the content includes any translated material, BMJ does not warrant the accuracy and reliability of the translations (including but not limited to local regulations, clinical guidelines, terminology, drug names and drug dosages), and is not responsible for any error and/or omissions arising from translation and adaptation or otherwise.

Open access This is an open access article distributed in accordance with the Creative Commons Attribution Non Commercial (CC BY-NC 4.0) license, which permits others to distribute, remix, adapt, build upon this work non-commercially, and license their derivative works on different terms, provided the original work is properly cited, appropriate credit is given, any changes made indicated, and the use is non-commercial. See: http://creativecommons.org/licenses/by-nc/4.0/.

\section{ORCID iD}

Christian Moro http://orcid.org/0000-0003-2190-8301

\section{REFERENCES}

1 Cameron JL, Halsted WS. Our surgical heritage. Ann Surg 1997;225:445-58.

2 Mason WTM, Strike PW. See one, do one, teach one--is this still how it works? A comparison of the medical and nursing professions in the teaching of practical procedures. Med Teach 2003;25:664-6.

3 Vozenilek J, Huff JS, Reznek M, et al. See one, do one, teach one: advanced technology in medical education. Acad Emerg Med 2004;11:1149-54.

4 Kapralos B, Uribe-Quevedo A, Dubrowski A. Immersive technologies for medical education. In: Lee NEncyclopedia of computer graphics and games. Cham: Springer International Publishing, 2017: 1-8.

5 Barsom EZ, Graafland M, Schijven MP. Systematic review on the effectiveness of augmented reality applications in medical training Surg Endosc 2016;30:4174-83.

6 McMillan K, Flood K, Glaeser R. Virtual reality, augmented reality, mixed reality, and the marine conservation movement. Aquat Conserv 2017;27:162-8.

7 Moro C, Birt J, Stromberga Z, et al. Virtual and augmented reality enhancements to medical and science student physiology and anatomy test performance: a systematic review and meta-analysis. Anat Sci Educ 2021;14:368-76.

8 Dyer E, Swartzlander BJ, Gugliucci MR. Using virtual reality in medical education to teach empathy. J Med Libr Assoc 2018;106:498-500.

9 Moro C, Štromberga Z, Raikos A, et al. The effectiveness of virtual and augmented reality in health sciences and medical anatomy. Anat Sci Educ 2017;10:549-59.

10 Moro C, Phelps C, Redmond P, et al. HoloLens and mobile augmented reality in medical and health science education: a randomised controlled trial. British Journal of Educational Technology 2021;52:680-94.

11 Craig E, Georgieva M. VR and AR: Driving a revolution in medical education \& patient care Educase Review: Educase, 2007. Available: https://er.educause.edu/blogs/2017/8/vr-and-ar-driving-a-revolutionin-medical-education-and-patient-care [Accessed March 2021]

12 Gardner J. Augmented and virtual reality in medicine: 6 applications we're keeping our eye on: Medteach Boston, 2016. Available: https:// medtechboston.medstro.com/blog/2016/05/24/16045 [Accessed March 2021].

13 World Health Organization. Dementia Geneva: who: World Health organization, 2020. Available: https://www.who.int/news-room/factsheets/detail/dementia [Accessed March 2021].

$14 \mathrm{Han}$ A, Kim TH, Hong H. Experiences of caregivers of people with dementia in a Korean dementia simulation program. Dementia 2020;19:2415-29.

15 World Health Organization. Dementia cases set to tripple by 2050 but still largely ignored Geneva: who: World Health organization, 2012. Available: https://www.who.int/mediacentre/news/releases/2012/ dementia 20120411/en/ [Accessed March 2021].

16 Beville PK. Virtual dementia tour helps sensitize health care providers. Am J Alzheimers Dis Other Demen 2002;17:183-90.

17 Adefila A, Graham S, Clouder L, et al. myShoes - the future of experiential dementia training? Journal of Mental Health Training 2016;11:91-101.

18 Gilmartin-Thomas JF-M, McNeil J, Powell A, et al. Impact of a virtual dementia experience on medical and pharmacy students' knowledge and attitudes toward people with dementia: a controlled study. $J$ Alzheimers Dis 2018;62:867-76.
19 Tullo E, Allan L. What should we be teaching medical students about dementia? Int Psychogeriatr 2011;23:1044-50.

20 Ball S, Bluteau P, Clouder L. myShoes: An immersive simulation of dementia. In: Watson CProceedings of the 10th International Conference on e-learning. Proceedings of the International Conference on e-Learning, 2015: 16-23.

21 Song Y, Hoben M, Weeks L, et al. Factors associated with the responsive behaviours of older adults living in long-term care homes towards staff: a systematic review protocol. BMJ Open 2019;9:e028416.

22 Feast A, Orrell M, Charlesworth G, et al. Behavioural and psychological symptoms in dementia and the challenges for family carers: systematic review. Br J Psychiatry 2016;208:429-34.

23 Kimzey M, Mastel-Smith B, Alfred D. The impact of educational experiences on nursing students' knowledge and attitudes toward people with Alzheimer's disease: a mixed method study. Nurse Educ Today 2016;46:57-63.

24 Page MJ, McKenzie JE, Bossuyt PM, et al. The PRISMA 2020 statement: an updated guideline for reporting systematic reviews. BMJ 2021;372:n71.

25 Centre for Reviews and Dissemination. Centre for reviews and dissemination York, England. Available: https://www.york.ac.uk/crd/ [Accessed March 2021].

26 Whittemore R, Knafl K. The integrative review: updated methodology. J Adv Nurs 2005;52:546-53.

27 Clark JM, Sanders S, Carter M, et al. Improving the translation of search strategies using the Polyglot search Translator: a randomized controlled trial. J Med Libr Assoc 2020;108:195-207.

28 Hong QN, Fàbregues S, Bartlett G, et al. The mixed methods appraisal tool (MMAT) version 2018 for information professionals and researchers. Education for Information 2018;34:285-91.

29 Hong QN, Gonzalez-Reyes A, Pluye P. Improving the usefulness of a tool for appraising the quality of qualitative, quantitative and mixed methods studies, the mixed methods appraisal tool (MMAT). J Eval Clin Pract 2018;24:459-67.

30 Gilmartin-Thomas JF-M, McNeil J, Powell A, et al. Qualitative evaluation of how a virtual dementia experience impacts medical and pharmacy students' self-reported knowledge and attitudes towards people with dementia. Dementia 2020;19:205-20.

31 Peng X, Wu L, Xie X, et al. Impact of virtual dementia tour on empathy level of nursing students: a quasi-experimental study. Int J Nurs Sci 2020;7:258-61.

32 Slater P, Hasson F, Gillen P, et al. Virtual simulation training: imaged experience of dementia. Int J Older People Nurs 2019;14:e12243.

33 Donahoe J, Moon L, VanCleave K. Increasing student empathy toward older adults using the virtual dementia tour. Journal of Baccalaureate Social Work2014;19:S-23-S-40.

34 Lorio AK, Gore JB, Warthen L, et al. Teaching dementia care to physical therapy doctoral students: a multimodal experiential learning approach. Gerontol Geriatr Educ 2017;38:313-24.

35 Werner D, Powell A, Clark R. Increasing empathy toward older adults with dementia: results of a dementia simulation. Journal of Baccalaureate Social Work2014;19:S-93-S-105.

36 Campbell D, Lugger S, Sigler GS, et al. Increasing awareness, sensitivity, and empathy for Alzheimer's dementia patients using simulation. Nurse Educ Today 2021;98:104764

37 Kimzey M, Mastel-Smith B, Seale A. Effects of dementia-specific education for nursing students. Nurse Educ 2019;44:338-41.

38 Kimzey M, Patterson J, Mastel-Smith B. Effects of simulation on nursing students' dementia knowledge and empathy: a mixed method study. Issues Ment Health Nurs 2021;42:274-9.

39 Mastel-Smith B, Kimzey M, Garner J, et al. Dementia care boot cAMP: interprofessional education for healthcare students. $J$ Interprof Care 2020;34:799-811.

40 Mastel-Smith B, Kimzey M, He Z. Dementia care education for nursing students. J Nurs Educ 2019;58:136-43.

41 Meyer K, James D, Amezaga B, et al. Simulation learning to train healthcare students in person-centered dementia care. Gerontol Geriatr Educ 2020:1-16.

42 Han A, Brown D. Experiences of caregivers in a dementia simulation program. J Soc Serv Res 2020;46:71-80.

43 Second Wind Dreams. Virtual dementia tour: second wind dreams, 2021. Available: https://www.secondwind.org/virtual-dementiatourreg.html [Accessed November 2020].

44 AGE-u-cate Traomomg Institute. Igniting change: AGE-u-cate training Institute, 2021. Available: https://ageucate.com/ [Accessed March 2021].

45 Kuhn D, King SP, Fulton BR. Development of the knowledge about memory loss and care (KAML-C) test. Am J Alzheimers Dis Other Demen 2005;20:41-9. 
46 Toye C, Lester L, Popescu A, et al. Dementia knowledge assessment tool version two: development of a tool to inform preparation for care planning and delivery in families and care staff. Dementia 2014;13:248-56.

47 Elvish R, Burrow S, Cawley R, et al. 'Getting to know me': the development and evaluation of a training programme for enhancing skills in the care of people with dementia in general Hospital settings. Aging Ment Health 2014;18:481-8.

48 O'Connor ML, McFadden SH. Development and psychometric validation of the dementia attitudes scale. Int J Alzheimers Dis 2010;2010:1-10.

49 Davis M. A multidimensional approach to individual differences in empathy. JSAS Catalog Sel Doc Psychol 1980;10:85-103.

50 Everson N, Levett-Jones T, Lapkin S. Comprehensive state empathy scale. University of Technology Sydney 2017.

51 Hojat M, Gonnella JS, Nasca TJ, et al. Physician empathy: definition, components, measurement, and relationship to gender and specialty. Am J Psychiatry 2002;159:1563-9.

52 Chardonnet J-R, Mirzaei MA, Mérienne F. Features of the postural sway signal as indicators to estimate and predict visually induced motion sickness in virtual reality. Int J Hum Comput Interact 2017;33:771-85.

53 Kennedy RS, Drexler J, Kennedy RC. Research in visually induced motion sickness. Appl Ergon 2010;41:494-503.
54 Bowman DA, Hodges LF, Allison D, et al. The educational value of an information-rich virtual environment. Presence 1999:8:317-31.

55 S-h W. Applying a 3D situational virtual learning environment to the real world business - an extended research in marketing. British Journal of Educational Technology 2012;43:411-27.

56 Moro C, Stromberga Z, Moreland A. Enhancing teaching in biomedical, health and exercise science with real-time physiological visualisations. In: Rea PMBiomedical visualisation. Cham: Springer International Publishing, 2020: 8. 1-11.

57 Bailenson JN, Yee N, Blascovich J, et al. The use of immersive virtual reality in the learning sciences: digital transformations of teachers, students, and social context. Journal of the Learning Sciences 2008:17:102-41.

58 Bowman DA, Sowndararajan A, Ragan ED. Higher levels of immersion improve procedure memorization performance. Proceedings of the 15th joint virtual reality Eurographics conference on virtual environments. Lyon, France: Eurographics Association; 2009: 121-8.

59 Webster R. Declarative knowledge acquisition in immersive virtual learning environments. Interactive Learning Environments 2016;24:1319-33.

60 Kolb D. Experiential learning: experience as the source of learning and development. In: Pearson education. 2nd edn, 2015. 